\title{
P03-10-34
}

Poster session

\section{Effect of ginsenoside Rb3 on the retinal vascular endothelial cells stimulated by high glucose}

\author{
Jing Han, Yan Wu
}

Beijing University of Chinese Medicine, China

OBJECTIVE To investigate the effect of ginsenoside $\mathrm{Rb} 3$, a compound from pseudo-ginseng, on migration and proliferation of the retinal vascular endothelial cells stimulated by high glucose. METHODS Retinal vascular endothelial cells were assigned into the normal group ( $5.5 \mathrm{mM}$ glucose), high glucose group ( $25 \mathrm{mM}$ glucose) and ginsenoside Rb3 group (25 mM glucose + ginsenoside Rb3 100, 150, $200 \mathrm{ug} / \mathrm{ml}$ respectively). After the cells were treated with different medium for three days, the CCK-8 assay, transwell migration assay, tube formation assay and BrdU proliferation assay were used to observe the effect of ginsenoside $\mathrm{Rb} 3$ on endothelial cells. Western blot method was used to examine the protein expression of the YAP1 and P-YAP1 in the retinal endothelial cells. RESULTS Compared with the normal group, the cell viability, the number of migrated cells, the tube length and the percentage of BrdU+ cells were increased in high glucose group $(\mathrm{P}<0.01)$; compared with the high glucose group, the cell viability, the number of migrated cells, the total length and the percentage of BrdU+ cells were inhibited by ginsenoside $\mathrm{Rb} 3(\mathrm{P}<0.05$ or $\mathrm{P}<0.01)$. The Western blot results showed that, in comparison to the normal group, the high glucose group showed higher expression of P-YAP1 and YAP1, $(\mathrm{P}<0.05)$; In comparison to the high glucose group, ginsenoside Rb3 inhibited the expression of P-YAP1 and YAP1 $(\mathrm{P}<0.05$ or $\mathrm{P}<0.01)$. CONCLUSION Ginsenoside Rb3 has a protective effect on the retinal vascular endothelial cells against high glucose, which is mediated by YAP1. 